\title{
Cardiac In-vivo Measurements Using Synthetic Transmit Aperture Ultrasound
}

Andresen, Henrik; Nikolov, Svetoslav; Hassager, Christian; Kjaergaard, Jesper; Jensen, Jørgen Arendt

Published in:

IEEE Ultrasonics Symposium

Link to article, DOI:

10.1109/ULTSYM.2006.286

Publication date:

2006

Document Version

Publisher's PDF, also known as Version of record

Link back to DTU Orbit

Citation (APA):

Andresen, H., Nikolov, S., Hassager, C., Kjaergaard, J., \& Jensen, J. A. (2006). Cardiac In-vivo Measurements Using Synthetic Transmit Aperture Ultrasound. In IEEE Ultrasonics Symposium (pp. 1115-1118). IEEE. https://doi.org/10.1109/ULTSYM.2006.286

\section{General rights}

Copyright and moral rights for the publications made accessible in the public portal are retained by the authors and/or other copyright owners and it is a condition of accessing publications that users recognise and abide by the legal requirements associated with these rights.

- Users may download and print one copy of any publication from the public portal for the purpose of private study or research.

- You may not further distribute the material or use it for any profit-making activity or commercial gain

- You may freely distribute the URL identifying the publication in the public portal 


\title{
Cardiac In-Vivo Measurements Using Synthetic Transmit Aperture Ultrasound
}

\author{
Henrik Andresen ${ }^{1,2}$, Svetoslav Nikolov ${ }^{1}$, Christian Hassager ${ }^{3}$, Jesper Kjærgaard ${ }^{3}$ and Jørgen Arendt Jensen ${ }^{1}$ \\ 1) Center for Fast Ultrasound Imaging, Ørsted•DTU, \\ Bldg. 348, Technical University of Denmark, DK-2800 Lyngby, Denmark. \\ 2) B-K Medical, Mileparken 34, DK-2730 Herlev, Denmark. \\ 3) Rigshospitalet, Department of Cardiology, Blegdamsvej 9, DK 2100 Copenhagen, Denmark.
}

\begin{abstract}
This paper investigates the feasibility of acquiring cardiac images using Synthetic Transmit Aperture (STA) ultrasound. Focusing in STA is done by beamforming all points in the image for every emission, creating a low-resolution image. The low-resolution images for each emission are summed, effectively achieving dynamic transmit and receive focusing. The purpose of this paper is to acquire in-vivo cardiac images using STA to investigate image quality and the effect of tissue motion.

For the in-vivo experiments, a $3 \mathrm{MHz}$ and a $3.5 \mathrm{MHz}$ transducers were used with 64 and 128 elements, respectively, together with the RASMUS experimental ultrasound scanner. Both transducers have a pitch of half a wavelength. To ensure an adequate signal-to-noise ratio, a $20 \mu$ s non-linear frequency modulated chirp and a 7-element de-focused virtual source were used for transmission. The number of virtual sources used in each scan sequence is equal to the number of transducer elements. A pulse repetition frequency of $4500 \mathrm{~Hz}$ was used, allowing a frame rate of 78 and 39 frames/s for the 64 and 128 element transducer.

As the heart walls move with a speed of up to $80 \mathrm{~mm} / \mathrm{s}$, a movement of the tissue of several wavelengths is possible during an STA scan sequence using 64 emissions. To investigate the possibility of reducing the susceptibility to tissue motion, a sparse scan sequence using only 9 virtual source emissions is used to reduce the required scan-time. A shorter scan sequence will reduce the tissue motion between the first and last emission, and allows a frame rate of up to 555 frames/s. The sparse sequence is interleaved with the full sequence to allow a better comparison between the two techniques.
\end{abstract}

A measurement of a point spread phantom shows a FWHM for the full scan sequences of $1.29 \mathrm{~mm}$ and $0.66 \mathrm{~mm}$ for the 64 and 128 element transducers, and an average side-lobe level of $-47.25 \mathrm{~dB}$ and $\mathbf{- 5 8 . 4 2} \mathrm{dB}$ respectively. The sparse scan sequences have a FWHM of $1.24 \mathrm{~mm}$ and $1.10 \mathrm{~mm}$ for the 64 and 128 element transducers, and an average side-lobe level of $\mathbf{- 3 9 . 4 8} \mathrm{dB}$

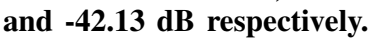

The four image sequences presented in this paper show a cross-section of the ventricle and atrium, where the walls of the ventricles and the atrioventricular valves are visible. The dynamic of the beating heart is visible during the recorded sequences. Distortions are seen in the images created by the full scan sequence, which are attributed to be caused by tissue motion. The images created by the sparse sequence show a reduced contrast, but also a reduction of the distortion caused by tissue motion.

\section{INTRODUCTION}

The use of synthetic transmit aperture (STA) for cardiac imaging offers both benefits and poses difficulties. STA allows for a better image quality, since it attains dynamic focusing in both transmit and receive. Faster imaging is also possible, as fewer emissions are necessary compared to traditional phased array (PA) imaging, which is often used for cardiac imaging due to the small footprint of a PA transducer. The drawbacks of STA are the susceptibility to artifacts caused by tissue motion, low signal-to-noise ratio (SNR), and the high computational demands. The tissue motion artifacts are caused by the sequential summation of several emissions.

Several people have performed research to estimate the effects of different types of tissue motion when doing STA [1]-[3]. They agree that lateral movement will not have a large impact on the resolution, where only a widening of the Point Spread Function (PSF) will occur. Axial movement will be more significant and the resulting PSF is heavily dependent on the emission sequence, tissue speed as well as the pulse repetition frequency. Nock and Trahey [2] estimates that movement up to $800 \mathrm{~mm} / \mathrm{s}$ does not cause substantial degradation of the beamformed PSF. As the heart wall moves between $50 \mathrm{~mm} / \mathrm{s}$ and $80 \mathrm{~mm} / \mathrm{s}$, this should be well within the limit.

The low SNR can be circumvented by using virtual source emissions as described in [4]-[6], and by using frequency modulation [7].

The purpose of this paper is to acquire in-vivo cardiac images using STA to investigate image quality and the effect of tissue motion. This paper presents the results from two cardiac in-vivo measurements using STA focusing with both full and sparse transmit aperture sampling. The acquisition method and techniques are described in Section II, the measurement setup and parameters are described in Section III, and the results are presented in Section IV. The paper concludes in Section V.

\section{THEORY}

In conventional ultrasound (US) beamforming the delay for each channel is based on the Time of Flight (ToF) from the center of the beam, to the desired beamformed point, and back to each element position. Dynamic focusing can be used in receive but not in transmit.

In STA imaging a spherical field is emitted, to circumvent the inability of conventional US to dynamically focus the beam in transmit. When emitting a spherical pulse, the exact ToF from an emitting element to an arbitrary point in the tissue 
and back to a given receive-element is given by

$$
t_{T o F}=\frac{1}{c}\left(\left|\vec{r}_{p}-\vec{r}_{m}\right|+\left|\vec{r}_{p}-\vec{r}_{n}\right|\right),
$$

where $\vec{r}_{p}$ is the beamformed point, $\vec{r}_{m}$ is the transmitting element and $\vec{r}_{n}$ is the receiving element.

By using (1) and summing the signal received for each transmit-receive pair, the algorithm for beamforming a point $\vec{r}_{p}$, giving the signal value $s\left(\vec{r}_{p}\right)$, is given by

$$
s\left(\vec{r}_{p}\right)=\sum_{m=1}^{M} \sum_{n=1}^{N} a_{n} g_{n, m}\left(t_{T o F}\right),
$$

where $M$ is the number of emissions, $N$ is the number of receiving elements, $g_{n, m}(t)$ is the complex signal from element number $n$ at time $t$ for emission $m$, and $a_{n}$ is the receive apodization of element number $n$. In practice, the image is created by beamforming all points for a single emission, resulting in a low resolution image. Each low resolution image is then summed to the resulting high resolution image.

By assuming the medium to be linear and stationary, dynamic focusing is attained everywhere in the medium, and not only at one depth per emission. The result is a narrower PSF overall in the image. It should be noted that conventional B-mode imaging and SA performs equivalently at the focal point of conventional imaging.

If only a subset of the transmit aperture is sampled, a decrease in the time for acquiring an image is possible at the expense of image quality. If the size of the sparse transmit aperture is the same as for a fully sampled aperture, the resolution is comparable to the fully sampled aperture according to theory, while the contrast will suffer due to changes in the effective aperture [8]. The reduction in acquisition time should also reduce the susceptibility to tissue motion, as the tissue will have less time to move from the first to the last emission.

\section{A. Virtual Sources}

SA suffers from a low SNR compared to conventional methods. A technique called virtual source (VS) emission has been proposed in [3]-[6], which allows several elements to create a waveform similar to that created by a single element. The timing of multiple elements will be chosen, so that the wavefront will seem to originate from the same position as with an emission originating from a single element behind or in front of the transducer. This is called either defocused or focused VS emission respectively. By using multiple transmit elements to form virtual sources, the SNR can be increased without decreasing the frame rate or resolution. The delays are calculated according to [6].

The wavefront created by the individual emissions will only be in phase within the angle $\theta_{a}$, denoted the acceptance angle given by

$$
\theta_{a}=\tan ^{-1} \frac{D_{a c t}}{2 F},
$$

where $D_{a c t}$ is the size of the active aperture, and $F$ is the depth of the virtual source relative to the transducer. Only points within this angle can be used to create the high resolution image.

\section{B. Frequency Modulated Emission}

Another method to increase the SNR of an SA emission is to emit more energy from each element. This can be attained by either increasing the transmit amplitude, or emitting a longer waveform. Both techniques have drawbacks though. By increasing the transmit amplitude a higher strain is put on the equipment. If the equipment has the potential of handling higher transmit amplitudes, FDA has given a limit for the peak amplitude, defined by the mechanical index. The disadvantage of using a chirp is the inability of imaging the near region, as simultaneous transmit and receive is not possible. A method for designing FM chirps specifically to the transducer has been proposed in [9] and used for the in-vivo studies. This method reduces the amount of energy absorbed by the transducer, allowing for longer and more effective chirps without overheating the transducer, and will be used here.

\section{Measurement Setup}

All measurement were performed on the RASMUS experimental ultrasound scanner. RASMUS is an abbreviation for Remotely Accessible Software configurable Multi-channel Ultrasound Sampling system, and was designed as a very flexible US system capable of transmitting arbitrary waveforms and storage of raw single channel data. A more detailed description is found in [10]. The system has 128 transmit channels and 64 channels in receive. Each transmit channel is able to store $128 \mathrm{kB}$ data for channel independent waveforms and each receive channel is able to store up to $256 \mathrm{MB}$ of data at a $40 \mathrm{MHz}$ sampling frequency. This allows for a large variety of transmission setups and approximately 3 seconds of continuous sampling of data for all receive channels.

To increase the SNR, both virtual sources and non-linear frequency modulation is used. The acceptance angle, given in (3), for the virtual sources is chosen to be $90^{\circ}$ to allow imaging of a large portion of the heart. The FM-chirp is designed to have axial side-lobes below $60 \mathrm{~dB}$, as well as a length of $20 \mu \mathrm{s}$. Combining the two techniques increase the penetration depth to $150 \mathrm{~mm}$ on phantoms, which have attenuation similar to tissue. The parameters for the two transducers and the imaging

TABLE I

TRANSDUCER AND MEASUREMENT PARAMETERS

\begin{tabular}{lll}
\hline Transducer name & $64 \mathrm{PA}$ & $128 \mathrm{PA}$ \\
\hline Transducer type & Phased array & Phased array \\
Number of transducer elements & 64 & 128 \\
Center frequency, $f_{0}$ & $3.0 \mathrm{MHz}$ & $3.5 \mathrm{MHz}$ \\
Transducer element pitch & $0.26 \mathrm{~mm}$ & $0.22 \mathrm{~mm}$ \\
Transducer element height & $13 \mathrm{~mm}$ & $15 \mathrm{~mm}$ \\
Elevation focus & $60 \mathrm{~mm}$ & $85 \mathrm{~mm}$ \\
\hline STA Scan Parameters & & \\
\hline Elements in virtual source & 9 & 9 \\
Emissions for full STA & 64 & 128 \\
$f_{\text {prf }}$ & 4500 & 4500 \\
VS Focusing F\# & $-\frac{1}{2}$ & $-\frac{1}{2}$ \\
FM-Chirp length & $20 \mu \mathrm{s}$ & $20 \mu \mathrm{s}$ \\
Scan depth & $150 \mathrm{~mm}$ & $150 \mathrm{~mm}$ \\
Receive apodization & Blackman & Blackman \\
\hline
\end{tabular}




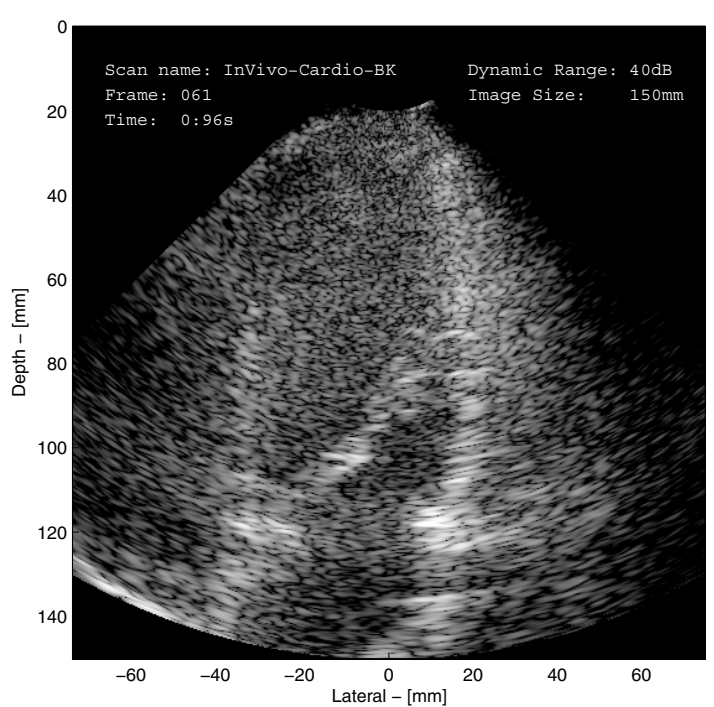

(a) PA 64 Full

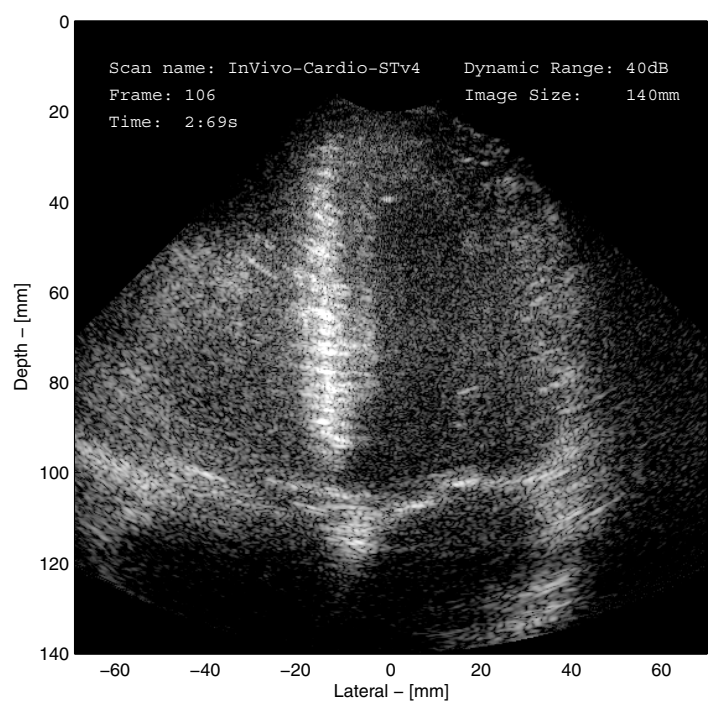

(c) PA 128 Full
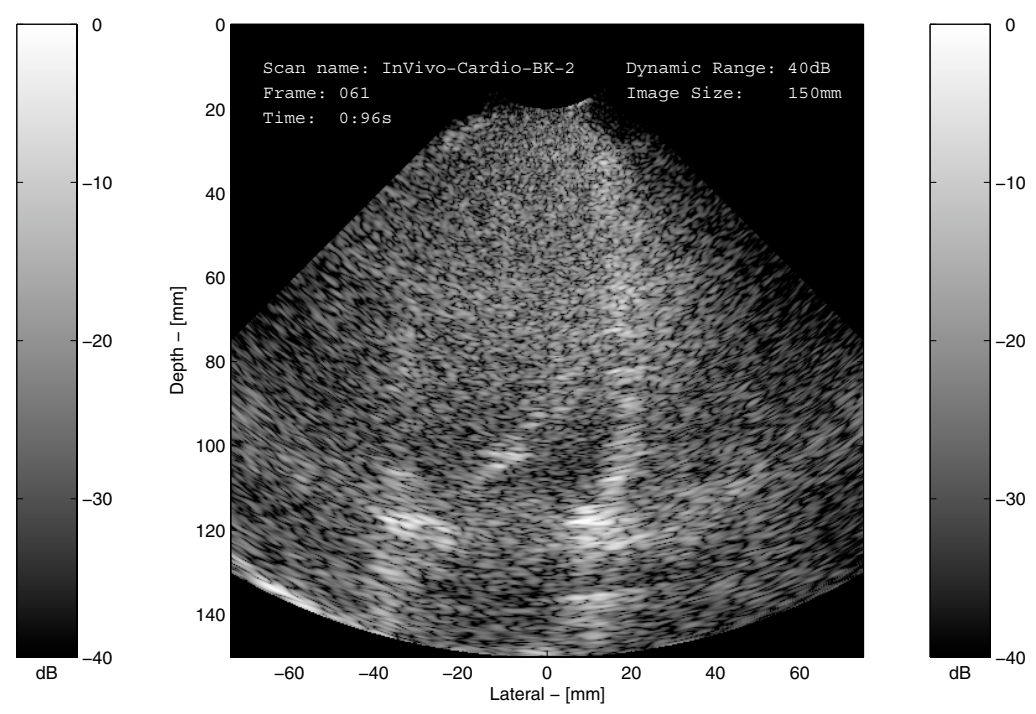

(b) PA 64 Sparse

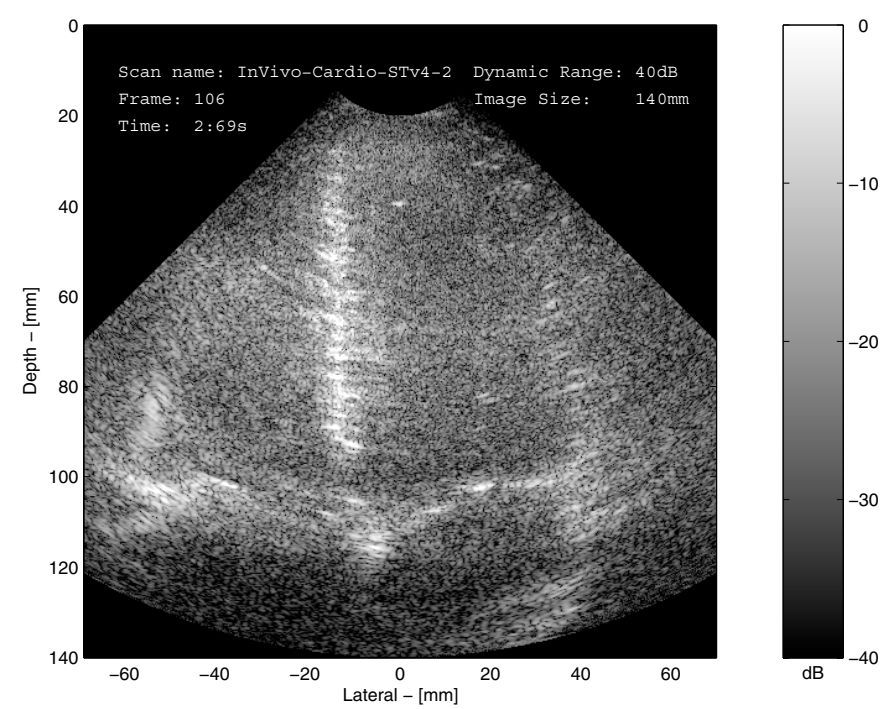

(d) PA 128 Sparse

Fig. 1. Figures (a) and (b) show STA images of the left ventricle made with the PA 64 transducer, with full and sparse aperture sampling respectively. Figure (c) and (d) show STA images of the left and right ventricle, with full and sparse aperture sampling respectively.

sequence are given in Table I. The negative $\mathrm{F}^{\#}$ in Table I denotes the virtual source is positioned behind the transducer giving a defocused field emission.

To allow for a more direct comparison between an in-vivo full STA scan and a sparse STA scan, where only a subset of the available sources are used, the sparse scan is embedded within the full STA scan. This is done by letting the first $K$-emissions make up the sparse array, and the remaining $K-N$-emissions are selected to sample the remaining transmit aperture. $K$ denotes the number of emissions used in the sparse sequence and $N$ is the total number of elements in the transducer. An example of the emissions sequence using a sparse scan of 4 elements for a 16 element transducer could be $[\mathbf{1}, \mathbf{6}, \mathbf{1 1}, \mathbf{1 6}, 2,3,4,5,7,8,9,10,12,13,14,15]$, where the numbers represent the emitting element, $K=4$ and
$N=16$. This emission pattern allows the full and the sparse data to be acquired simultaneously without the need for two separate imaging sequences.

\section{RESUlts}

The atrium and ventricle of the heart were scanned with two different transducers, utilizing a full STA imaging sequence as well as an embedded sparse imaging sequence. The first scanning was performed with the 64 PA transducer, and a frame of the scan sequence for $t=0.96 \mathrm{~s}$ is shown in Fig. 1(a). The left ventricle is visible along with the heart walls and the valve separating the atrium from the ventricle. Part of the left atrium is visible as well. The image is shown with a dynamic range of $40 \mathrm{~dB}$. This imaging mode allows for a frame rate of $70 \mathrm{~Hz}$, compared to the $33 \mathrm{~Hz}$ for a phased array when using the angular sampling criteria given in [11]. 
The contrast in the image is not very good and the individual features of the heart are not clearly distinct. As this blurring might originate from tissue motion, an image was beamformed using only the sparse sequence as described in Section III. The image created from the sparse sequence is shown in Fig. 1(b). The most immediate difference is the sparse sequence has a reduced contrast. The sparse sequences should be less susceptible to tissue motion, as the maximum movement of the heart wall is lowered from $2.58 \lambda$ to $0.36 \lambda$ when using only 9 emissions instead of 64 emission. A center frequency of 3.5 $\mathrm{MHz}$ is used and the velocity of the heart wall is assumed to be $80 \mathrm{~mm} / \mathrm{s}$ [12]. This indicates the reduced contrast overshadows any improvement from the reduced scan time.

The 128 PA transducer was used to increase the image quality as the transducer has a higher bandwidth and larger aperture size. The scan is taken from the same angle as the first scan, but is tilted to allow the right atrium and ventricle to be within the imaging area. Both the full and the sparse aperture scan are shown in Fig. 1(c) and 1(d). The main difference is a much higher contrast between the blood filled chambers and the cardiac walls. The sparse scan suffers from a reduction in contrast, which was also seen for the PA 64 transducer.

To investigate the cause of the reduced contrast in the images, a phantom containing a single point scatterer was scanned with the same imaging sequence. The measured point spread function (PSF) for the full and the sparse aperture sequence is seen in Fig. 2. The PSF has a full width at half maximum (FWHM) of $0.66 \mathrm{~mm}$ and $1.10 \mathrm{~mm}$ for the full and the sparse sequence respectively, and a mean side-lobe level of $-58.24 \mathrm{~dB}$ and $-42.14 \mathrm{~dB}$. Both the resolution and the contrast decrease. The contrast is on average degraded by $>15 \mathrm{~dB}$ for the $128 \mathrm{PA}$ transducer. An identical measurement for the 64 PA transducer was made showing a FWHM of $1.29 \mathrm{~mm}$ and $1.24 \mathrm{~mm}$ and side-lobes of $-47.24 \mathrm{~dB}$ and $-42.13 \mathrm{~dB}$ for the full and sparse aperture sequence respectively.

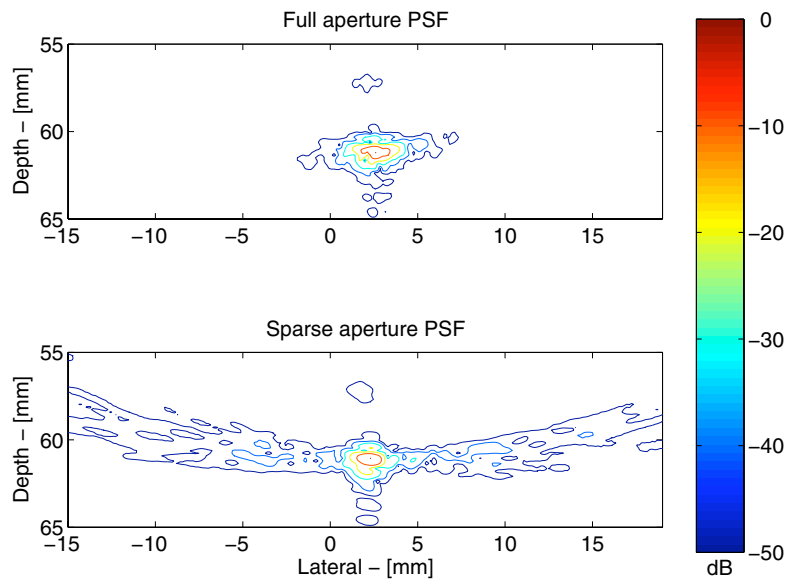

Fig. 2. PSF og a small diamond for a full and a sparse aperture scan using the PA 64 transducer.

\section{CONCLUSION}

In-vivo cardiac imaging is possible using STA without motion compensation. Images were acquired for a 128 and a 64 element transducer with both full and sparse transmit aperture sampling. In both cardiac scans, it was possible to distinguish the heart wall as well as the heart valve separating the ventricle and the atrium. The images from the 128 element transducer had an increase in contrast compared to the 64 element transducer as expected.

A sparse scan was inlaid with the normal scan, which only used 9 elements. The sparse sequences seems to indicate that the side-lobe level for the PSF is very important in obtaining high quality cardiac scans. The scan sequences should thus be optimized for reduced side-lobe levels before the effects of tissue motion can be seen. Further studies are required to optimize the scan sequences and hereby increase the image quality. Also, the effect different apodization and motion compensation techniques should be investigated.

\section{ACKNOWLEDGMENT}

This work was supported by grant 9700883, 9700563 and 26-04-0024 from the Danish Science Foundation and by B-K Medical, Denmark.

\section{REFERENCES}

[1] C. R. Hazard and G. R. Lockwood. Effects of motion artifacts on a synthetic aperture beamformer for real-time $3 \mathrm{~d}$ ultrasound. In Proc. IEEE Ultrason. Symp., pages 1221-1224, 1999.

[2] L. F. Nock and G. E. Trahey. Synthetic receive aperture imaging with phase correction for motion and for tissue inhomogenities - part I: basic principles. IEEE Trans. Ultrason., Ferroelec., Freq. Contr., 39:489-495, 1992.

[3] S. I. Nikolov. Synthetic aperture tissue and flow ultrasound imaging. PhD thesis, Ørsted $\bullet D T U$, Technical University of Denmark, 2800, Lyngby, Denmark, 2001.

[4] M. H. Bae and M. K. Jeong. A study of synthetic-aperture imaging with virtual source elements in B-mode ultrasound imaging systems. In IEEE Trans. Ultrason., Ferroelec., Freq. Contr., volume 47, pages 1510-1519, 2000.

[5] M. Karaman, P. C. Li, and M. O’Donnell. Synthetic aperture imaging for small scale systems. IEEE Trans. Ultrason., Ferroelec., Freq. Contr, 42:429-442, 1995.

[6] S. I. Nikolov and J. A. Jensen. Virtual ultrasound sources in highresolution ultrasound imaging. In Proc. SPIE - Progress in biomedical optics and imaging, volume 3, pages 395-405, 2002.

[7] K. L. Gammelmark and J. A. Jensen. Multielement synthetic transmit aperture imaging using temporal encoding. IEEE Trans. Med. Imag., 22(4):552-563, 2003.

[8] G. R. Lockwood, J. R. Talman, and S. S. Brunke. Real-time 3-D ultrasound imaging using sparse synthetic aperture beamforming. IEEE Trans. Ultrason., Ferroelec., Freq. Contr., 45:980-988, 1998.

[9] F. Gran and J. A. Jensen. Designing non-linear frequency modulated signals for medical ultrasound imaging. In Proc. IEEE Ultrason. Symp., page Accepted, 2006.

[10] J. A. Jensen, O. Holm, L. J. Jensen, H. Bendsen, S. I. Nikolov, B. G. Tomov, P. Munk, M. Hansen, K. Salomonsen, J. Hansen, K. Gormsen, H. M. Pedersen, and K. L. Gammelmark. Ultrasound research scanner for real-time synthetic aperture image acquisition. IEEE Trans. Ultrason., Ferroelec., Freq. Contr., 52 (5):881-891, May 2005.

[11] K. L. Gammelmark and J. A. Jensen. Experimental study of convex coded synthetic transmit aperture imaging. In Proc. IEEE Ultrason. Symp., pages 1573-1576, 2002.

[12] C. R. Hazard and G. R. Lockwood. Theoretical assessment of a synthetic aperture beamformer for real-time 3-D imaging. IEEE Trans. Ultrason., Ferroelec., Freq. Contr., 46:972-980, 1999. 